variety of stakeholders to identify innovations and promising approaches to transform the landscape and catalyse the field of global mental health, including advances in implementation research, novel ways to deliver mental health treatments, the use of technology and addressing the social determinants of mental health. Mental health in the SDGs is an indication of a new dawn for the global development agenda, but it will require an integrated, inter-sectoral scientific collaboration to bring to light the true importance of mental health for sustainable global development.

\section{References}

Baingana F. \& Mangen P. O. (2011) Scaling up of mental health and trauma support among war affected communities in northern Uganda: lessons learned. Intervention, 9, 291-303.

Bass J. K., Annan J., Mcivor Murray S., et al (2013) Controlled trial of psychotherapy for Congolese survivors of sexual violence. New England Journal of Medicine, 368, 2182-2191.

Bolton P., Bass J., Neugebauer R., et al (2003) Group interpersonal psychotherapy for depression in rural Uganda: a randomized controlled trial. Journal of the American Medical Association, 289, 3117-3124.

Chisholm D., Sweeny K., Sheehan P., et al (2016) Scaling-up treatment of depression and anxiety: a global return on investment analysis. The Lancet Psychiatry, 3, 415-424.

Collins P. Y. \& Saxena S. (2016) Action on mental health needs global cooperation. Nature, 532, 25.

De Silva M. J. \& Ryan G. (2016) Global mental health in 2015: 95\% implementation. The Lancet Psychiatry, 3, 15-17.
Detlev V. (2015) Does the United Nations care about mental health? British Journal of Psychiatry, 206, 289-296.

Petersen I., Evans-Lacko S., Semrau M., et al (2016) Promotion prevention and protection: interventions at the population- and community-levels for mental, neurological and substance use disorders in low- and middle-income countries. International Journal of Mental Health Systems, 10, 1.

Rahman A., Malik A., Sikander S., et al (2008) Cognitive behaviour therapy-based intervention by community health workers for mothers with depression and their infants in rural Pakistan: a cluster-randomised controlled trial. Lancet, 372, 902-909.

Sin N. L. \& Dimatteo M. R. (2014) Depression treatment enhances adherence to antiretroviral therapy: a meta-analysis. Annals of Behavioral Medicine, 47, 259-269.

Ventevogel P., Van De Put W., Faiz H., et al (2012) Improving access to mental health care and psychosocial support within a fragile context: a case study from Afghanistan. PLoS Medicine, 9, e1001225.

Whiteford H. A., Ferrari A. J., Degenhardt L., et al (2015) The global burden of mental, neurological and substance use disorders: an analysis from the global burden of disease study 2010. PLoS One, 10 e0116820.

World Health Organization (WHO) (2001) The World Health Report 2001: Mental Health: New Understanding, New Hope. World Health Organization.

World Health Organization (WHO) (2010) Mental Health Gap Action Programme: MhGAP Intervention Guide for Mental, Neurological and Substance Use Disorders in Non-specialized Health Settings: Version 1.0, pp. 86-94. World Health Organization.

World Health Organization (WHO) (2015) World Mental Health Atlas 2014. World Health Organization.

\title{
THEMATIC \\ Inclusion of mental health in global economic development
}

\author{
Chee H. Ng, ${ }^{1}$ Maureen M. Goodenow, ${ }^{2}$ Andrew J. Greenshaw, ${ }^{3}$ Phil Upshall ${ }^{4}$ \\ and Raymond W. Lam ${ }^{5}$
}

${ }^{1}$ Department of Psychiatry and Asia-Australia Mental Health University of Melbourne, Melbourne, Australia, email: cng@unimelb.edu.au

${ }^{2}$ National Institutes of Health, Bethesda, Maryland, USA

${ }^{3}$ Department of Psychiatry, University of Alberta, Edmonton, Canada

${ }^{4}$ Mood Disorders Society of Canada, City of Guelph, Ontario Canada

${ }^{5}$ Department of Psychiatry, University of British Columbia Vancouver, Canada

Conflicts of interest. None.

doi:10.1192/bji.2017.23

\begin{abstract}
The APEC Mental Health Roadmap has a vision to strengthen mental health and reduce the economic impact of mental illness in the Asia Pacific. To facilitate its implementation, the APEC Digital Hub will heighten exchange and dissemination of best practices in Asia Pacific mental health partnerships, and increase multi-sectoral recognition to invest in mental health to support economic growth.
\end{abstract}

The Asia-Pacific Economic Cooperation (APEC) forum is a regional inter-governmental economic organisation of 21 Pacific Rim member economies $^{1}$ that aims to promote sustainable

${ }^{1}$ APEC economies include Australia; Brunei Darussalam; Canada; Chile; China; Hong Kong, China; Indonesia; Japan; Republic of Korea; Malaysia; Mexico; New Zealand; growth, free trade and economic integration. The APEC region includes the world's three largest economies and is home to about 2.8 billion people from highly diverse cultures, representing approximately $57 \%$ of world gross domestic product. APEC members deal with important new challenges to the region's economic well-being, including disaster resilience, pandemics, climate change and terrorism. APEC committees and working groups provide strategic policy recommendations to APEC economic leaders (heads of state) and ministers, who meet annually to set the overarching goals and initiatives. The working groups are then tasked with implementing these initiatives.

Papua New Guinea; Peru; the Philippines; the Russian Federation; Singapore; Chinese Taipei; Thailand; the United States; and Viet Nam. 
(c) The Authors 2018. This is an Open Access article, distributed under the terms of the Creative Commons Attribution-

NonCommercial-NoDerivatives

licence (http://creativecommons. org/licenses/by-nc-nd/4.0/), which permits non-commercial re-use, distribution, and reproductio any medium, provided the or ginal work is unaltered and is properly cited. The written permission of Cambridge University Press must be obtained for commercial re-use or in order to create a derivative work.
For the first time in APEC's 25-year history, mental health was raised in the 2014 APEC leaders declaration, in recognition of the central role of health in achieving sustainable development (APEC, 2014a). APEC ministers endorsed the landmark APEC Roadmap to Promote Mental Wellness in a Healthy Asia Pacific (2014-2020) (APEC, 2014b) and called for the implementation of the Roadmap's goals, which are aligned with the Healthy Asia Pacific 2020 initiative to develop sustainable health systems. The Roadmap's activities complement the World Health Organization's (WHO) Mental Health Action Plan (2013-2020) (WHO, 2013), and are consistent with the Commonwealth of Nations' focus on the economic and social inclusion of mental health, particularly the economic empowerment and social inclusion of people who have experienced mental health challenges $(\mathrm{Ng}, 2013)$.

The World Economic Forum notes that chronic disease will cost the global economy more than US $\$ 47$ trillion between 2010 and 2030, with one-third (US $\$ 16$ trillion) of this cost attributed to mental disorders (Bloom et al, 2011). Mental disorders are a leading cause of years lived with disability and account for $37 \%$ of all healthy life years lost to chronic disease. Despite this challenge, annual spending worldwide on mental health remains less than US $\$ 2$ per person. Consequently, a multilateral economic forum taking action on mental health and its socioeconomic effects on a regional scale is visionary and highly significant.

The WHO has outlined the economic and social effects of mental and psychosocial disabilities, including homelessness, poor education and health outcomes, high rates of unemployment and poverty (WHO, 2010). Obstacles to care for and recovery from mental disorders, including social stigma, shortages of specialists, insufficient training of primary health professionals, inadequate prevention programmes, and limited access to quality care or effective medications, compound the growing pressure mental health challenges place on over-stretched health systems, particularly in APEC low- and middleincome economies.

The APEC leaders recognise that mental health is critical to overall health, social and economic participation, workplace productivity and sustainable economic growth in the Asia Pacific region. Leaders have noted that inaction by APEC economies to prioritise and strengthen mental health will result in costs that impede the achievement of economic development. Member economies acknowledge the significant economic benefits of a mentally healthy population, and that prevention of and recovery from mental disorders is possible through affordable and effective treatments and care. Swift economic changes, ageing populations and natural disasters raise special mental health challenges for APEC economies that require proactive and concerted responses. There are significant opportunities for APEC economies to strengthen mental health through heightened investment and the facilitation of multi-lateral, multi-sectoral collaborations and public-private partnerships.

The Roadmap's focus on innovation, facilitating public-private engagement and building on APEC platforms aligns with broader development strategies within the region, including promoting best practices and examples of novel collaborations in global mental health. Supporting the implementation of APEC's Roadmap will make an important contribution to sustainable economic growth and poverty reduction, in line with the United Nations' sustainable development goals (SDGs), especially SDG 3.4, the health and well-being target (Sustainable Development, 2015). The Roadmap builds on strategic opportunities in the region to strengthen leadership in innovative and effective mental healthcare interventions. To this end, APEC has a formed a strategic partnership with the World Bank Group (WBG) and the WHO at the WBG and International Monetary Fund Spring Meeting 2016 (World Bank, 2016).

In November 2016, APEC ministers welcomed efforts to campaign for mental health, including the development of projects under the newly launched APEC Digital Hub for Mental Health (APEC, 2016a). The mission of the Digital Hub (https://mentalhealth.apec.org) is to enhance awareness, share information and experiences, develop customized curricula, and facilitate the identification and implementation of evidence- and practice-based models in multi-lateral and diverse public-private partnerships. A key aspect of the Digital Hub is a commitment to improve mental health service access and to fully engage people with lived experience in all aspects of service delivery. The Digital Hub will serve as a regional incubator of new ideas and practices, a platform for scaling-up and the focal point in APEC's work to implement the Roadmap.

The host institution, the University of British Columbia, in collaboration with the University of Alberta and the Mood Disorders Society of Canada (MDSC), serves as the coordinating centre supporting the Digital Hub through a network of core partner institutions that provide resources, services, advice and technical expertise to promote an interactive, web-based platform to all APEC stakeholders. A unique strength is the experience of MDSC in actively engaging with all community mental health stakeholders in the development of shared mental healthcare in Canada (www.ccmhi.ca) and the establishment of the Canadian Depression Research and Intervention Network (http://cdrin.org) - both activities are government funded. MDSC was principally responsible for the development of curricula to facilitate the full engagement of people with lived experience in all aspects of mental health service and research (http://cdrin.org/lived-experience).

Following a strategic needs assessment, the common priority areas of mental health development identified by APEC economies include: workplace wellness and resilience; integration with primary 
care and community settings; advocacy and public awareness; mental wellness for indigenous communities, vulnerable communities and children; data collection and standardisation; and disaster resilience and trauma. Within these areas, the Digital Hub will facilitate interactive training modules, map mental health stakeholders, and showcase novel research on the effects of mental health, as well as the cost-benefit analysis of investment for interventions. Based on the strategic needs assessments, five recommendations are proposed (APEC, 2016b):

1. to advance an APEC-wide strategy that includes legislative bodies to advocate for mental health, as well as to enhance public awareness to reduce social stigma;

2. to establish expert working committees and novel partnerships through the APEC Digital Hub for Mental Health to address each of the common priority areas;

3. to strengthen linkages with the APEC Business Advisory Council and expert organisations, particularly in the promotion and strengthening of workplace mental health;

4. to build linkages with the APEC Emergency Preparedness Working Group in disaster resilience and trauma activities; and

5. to develop APEC resources centred on the linkage between mental health and economic growth/sustainability.

Implementation of the APEC Roadmap and Digital Hub will heighten exchange and dissemination of best practices and innovations in Asia-Pacific mental health partnerships. The Hub provides an unprecedented opportunity to enhance recognition among the highest government leaders, health and non-health officials, institutions and organisations, as well as the public, of the importance of strengthened and strategic investment in mental health to support economic growth. APEC has an aspirational vision to strengthen mental health and reduce the economic effects of mental illness in the Asia Pacific region. Success of the venture will become an exemplar of the positive influence that multi-stakeholder collaboration and public-private partnerships can have to improve mental wellness for millions across a wide diversity of settings and cultures.

\section{References}

APEC (2014a) APEC Ministerial Endorsement. APEC. Available at http://www.apec.org/Meeting-Papers/Annual-Ministerial-Meetings/ 2014/2014_amm.aspx.

APEC (2014b) APEC Roadmap to Promote Mental Wellness in a Healthy Asia Pacific (2014-2020). APEC. Available at http://mddb. apec.org/Documents/2014/MM/AMM/14_amm_014.pdf.

APEC (2016a) APEC Ministerial Meeting 2016. APEC. Available at http://www.apec.org/Meeting-Papers/Annual-Ministerial-Meetings/ 2016/2016_amm.aspx.

APEC (2016b) APEC Mental Health Initiative. Report on Strategic Needs in Mental Health. July 2016. APEC. Available at https:// mentalhealth.apec.org/sites/default/files/

APEC_Report_on_Strategic_Needs_in_Mental_Health_Final-3.pdf.

Bloom D. E., Cafiero E. T., Jané-Llopis E., et al (2011) The Global Economic Burden of Noncommunicable Diseases. World Economic Forum. Available at http://apps.who.int/medicinedocs/documents/ s18806en/s18806en.pdf.

Ng C. (2013) Mental Health: Towards Economic and Social Inclusion: A Report to the Commonwealth Secretariat. Commonwealth Secretariat.

Sustainable Development (2015) Transforming Our World: The 2030 Agenda for Sustainable Development. United Nations. Available at https://sustainabledevelopment.un.org/post2015/transformingourworld.

World Bank (2016) Making Mental Health a Global Priority 2016. World Bank Group. Available at http://pubdocs.worldbank.org/en/39117146539 3131073/0602-SummaryReport-GMH-event-June-3-2016.pdf.

World Health Organization (2010) Mental Health and Development. WHO

World Health Organization (2013) Comprehensive Mental Health Action Plan (2013-2020). WHO. Available at http://www.who.int/ mental_health/action_plan_2013/en/.

\title{
THEMATIC \\ Mental health and integration PAPER in Asia Pacific
}

\author{
Chee $\mathrm{H} . \mathrm{Ng}$
}

Department of Psychiatry, University of Melbourne, Australia; email cng@unimelb. edu.au

Acknowledgements. The report 'Provision for supporting people with mental illness: A comparison of 15 Asia-Pacific countries' (2016) was prepared and written by The Economist Intelligence Unit (EIU) and sponsored by Janssen Asia Pacific.
This brief report examines the extent to which community-based treatment and integration support are provided for people living with mental illness across 15 selected Asia-Pacific economies. Some of the key findings are discussed in light of the diversity of economies and cultural contexts.

\section{Background}

The 2010 Global Burden of Disease Study estimated that mental and substance use disorders accounted for $7.4 \%$ of all disability-adjusted life years (DALYs) globally, an increase of $37.6 \%$ over the preceding 20 years (Whiteford et al, 2013). Mental and substance use disorders were the leading cause of years lost due to disability (YLDs) worldwide. Asia Pacific is a region characterised by rapid changes in economic and technological 